\title{
On Polish Groups of Finite Type
}

\author{
by
}

\author{
Hiroshi ANDO and Yasumichi Matsuzawa
}

\begin{abstract}
Sorin Popa initiated the study of Polish groups which are embeddable into the unitary group of a separable finite von Neumann algebra. Such groups are called of finite type or said to belong to the class $\mathscr{U}_{\text {fin }}$. We give necessary and sufficient conditions for Polish groups to be of finite type, and construct examples of such groups for $\mathrm{I}_{\infty}$ and $\mathrm{II}_{\infty}$ von Neumann algebras. We also discuss permanence properties of finite type groups under various algebraic operations. We close the paper with some questions concerning such groups.
\end{abstract}

2010 Mathematics Subject Classification: Primary 46L10; Secondary 54H11.

Keywords: bi-invariant metric, class $\mathscr{U}_{\text {fin }}$, finite type group, Polish group, positive definite function, SIN-group, $\mathrm{II}_{1}$ factor.

\section{$\S 1$. Introduction}

In this paper we consider the following problem. Denote by $\mathcal{U}(M)$ the unitary group of a von Neumann algebra $M$.

Problem. Determine a necessary and sufficient condition for a Polish group $G$ to be isomorphic as a topological group to a strongly closed subgroup of some $\mathcal{U}(M)$, where $M$ is a separable finite von Neumann algebra.

S. Popa defined a Polish group to be of finite type if it has this embedding property. Denote by $\mathscr{U}_{\text {fin }}$ the class of all finite type Polish groups. He initiated the study of this class in an attempt to enrich the study of rapidly developing cocycle

Communicated by H. Okamoto. Received May 28, 2011. Revised July 1, 2011.

H. Ando: Department of Mathematical Sciences, University of Copenhagen, Universitetsparken 5, 2100 København $\varnothing$, Denmark, and Research Institute for Mathematical Sciences,

Kyoto University, Kyoto, 606-8502, Japan;

e-mail: andonuts@kurims.kyoto-u.ac.jp

Y. Matsuzawa: Mathematisches Institut, Universität Leipzig, Johannisgasse 26, 04103, Leipzig, Germany, and Department of Mathematics, Hokkaido University,

Kita 10, Nishi 8, Kita-ku, Sapporo, 060-0810, Japan

e-mail: matsuzawa@math.sci.hokudai.ac.jp

(C) 2012 Research Institute for Mathematical Sciences, Kyoto University. All rights reserved. 
superrigidity theory (cf. $[8,16,18])$. In particular, he proposed in [18] the problem of studying and characterizing the class $\mathscr{U}_{\text {fin }}$.

Secondly, this problem is motivated by our previous work [1] on infinitedimensional Lie algebras associated with such groups: Let $M$ be a finite von Neumann algebra on a Hilbert space $\mathcal{H}$. Let $G$ be a strongly closed subgroup of $\mathcal{U}(M)$ and $\bar{M}$ be a set of all densely defined closed operators on $\mathcal{H}$ which are affiliated to $M$. It is known that the set

$$
\operatorname{Lie}(G):=\left\{A^{*}=-A \in \bar{M} ; e^{t A} \in G \text { for all } t \in \mathbb{R}\right\}
$$

is a complete topological Lie algebra with respect to the strong resolvent topology (see also the related work of D. Beltiţă [3]). Since these Lie algebras turn out to be non-locally convex in general when $M$ is nonatomic, they are quite exotic as Lie algebras and their properties are poorly known. Therefore it would be interesting to find non-trivial examples of such groups.

We give an answer in Theorem 2.7 to the above Problem with the aid of positive definite functions on groups and their GNS representations, and characterize locally compact groups or amenable Polish groups of finite type via compatible bi-invariant metrics in Proposition 2.20 and Theorem 2.22 (the former is known, but we give a new proof). Combined with Popa's result [18], Theorem 2.7 gives a necessary and sufficient condition for a Polish group to be isomorphic to a closed subgroup of the unitary group of a separable $\mathrm{II}_{1}$ factor. We then give examples of Polish groups of finite type using noncommutative integration of E. Nelson [17]. Finally we discuss some hereditary properties of finite type groups and pose some questions concerning Polish groups of finite type.

Notation. In this paper we often say a von Neumann algebra $M$ is separable if it has a separable predual, especially when the Hilbert space on which $M$ acts is implicit. This is known to be equivalent to the condition that $M$ has a faithful representation on a separable Hilbert space. We denote by $\operatorname{Proj}(M)$ the lattice of all projections in $M$. A von Neumann algebra is said to be finite if it admits no nonunitary isometry. When we consider a group $G$, its identity is denoted by $e_{G}$. However, we also use 1 for the identity when we consider a concrete subgroup of the unitary group of a von Neumann algebra. We always regard the unitary group of a von Neumann algebra as a topological group with the strong operator topology.

\section{$\S 2$. Polish groups of finite type and their characterization}

In this section, we characterize Polish groups of finite type via positive definite functions. We then characterize when locally compact groups or amenable Polish 
groups are of finite type via compatible bi-invariant metrics. To this end, we review the notions of SIN-groups, bi-invariant metrics and unitary representability.

\section{$\S 2.1$. Polish groups of finite type}

Recall that a Polish space is a separable completely metrizable topological space, and a Polish group is a topological group whose topology is Polish.

We now introduce finite type groups after Popa [18].

Definition 2.1. A Hausdorff topological group is called of finite type if it is isomorphic as a topological group to a closed subgroup of the unitary group of a finite von Neumann algebra.

Remark 2.2. Popa [18] requires the topological group of finite type to be Polish, whereas our definition of finiteness does not require any countability. We will show in Theorem 2.7 that a Polish group $G$ of finite type in our sense satisfies Popa's definition of finite type group. That is, $G$ is isomorphic to a closed subgroup of the unitary group of a finite von Neumann algebra acting on a separable Hilbert space.

All second countable locally compact Hausdorff groups and the unitary group of a von Neumann algebra acting on a separable Hilbert space are Polish groups. Furthermore, separable Banach spaces are Polish groups when viewed as additive groups. We denote the class of all Polish groups of finite type by $\mathscr{U}_{\text {fin }}$.

Note that since a von Neumann algebra is finite if and only if its unitary group is complete with respect to the left uniform structure, Polish groups of finite type are necessarily complete. Thus we have the following simple consequence.

Proposition 2.3. The unitary group of a von Neumann algebra $M$ acting on a separable Hilbert space is of finite type if and only if $M$ is finite.

Other examples of Polish groups of finite type are given later.

\section{$\S 2.2$. Positive definite functions}

A complex valued function $f$ on a Hausdorff topological group $G$ is called positive definite if for all $g_{1}, \ldots, g_{n} \in G$ and for all $c_{1}, \ldots, c_{n} \in \mathbb{C}$,

$$
\sum_{i, j=1}^{n} \bar{c}_{i} c_{j} f\left(g_{i}^{-1} g_{j}\right) \geq 0 .
$$

If a complex valued function $f$ on $G$ is invariant under inner automorphisms, that is,

$$
f\left(h g h^{-1}\right)=f(g), \quad \forall g, h \in G,
$$

then $f$ is called a class function. 
It is well-known that there is a one-to-one correspondence between the set of all continuous positive definite functions on a topological group and the set of unitary equivalence classes of cyclic unitary representations of the group. More precisely, for each continuous positive definite function $f$ on a topological group $G$, there exists a triple $\left(\pi_{f}, \mathcal{H}_{f}, \xi_{f}\right)$ consisting of a cyclic unitary representation $\pi_{f}$ in a Hilbert space $\mathcal{H}_{f}$ and a cyclic vector $\xi_{f}$ in $\mathcal{H}_{f}$ such that

$$
f(g)=\left\langle\xi_{f}, \pi_{f}(g) \xi_{f}\right\rangle, \quad g \in G,
$$

and this triple is unique up to unitary equivalence. The triple is called the GNS triple associated to $f$. Note that if $G$ is separable, then so is $\mathcal{H}_{f}$.

The GNS triple is of the following form for each continuous positive definite class function.

Lemma 2.4. Let $f$ be a continuous positive definite class function on a topological group $G$ and $(\pi, \mathcal{H}, \xi)$ be its GNS triple. Then the von Neumann algebra $M$ generated by $\pi(G)$ is finite and the linear functional

$$
\tau(x):=\langle\xi, x \xi\rangle, \quad x \in M,
$$

is a faithful normal tracial state on $M$. In particular $M$ is countably decomposable.

Proof. It is clear that $\tau$ is a normal state on $M$. Since $f$ is a class function, it is easy to see that $\tau$ is tracial on the strongly dense ${ }^{*}$-subalgebra of $M$ spanned by $\pi(G)$. Therefore by normality, $\tau$ is tracial on $M$. Hence we have only to check the faithfulness of $\tau$. Assume $\tau\left(x^{*} x\right)=0$. Since $\tau$ is a trace, we have

$$
\|x \pi(g) \xi\|^{2}=\tau\left(\pi(g)^{*} x^{*} x \pi(g)\right)=0
$$

for all $g \in G$. By the cyclicity of $\xi, x$ must be 0 .

Example 2.5 (I. J. Schoenberg [19]). Let $\mathcal{H}$ be a complex Hilbert space. Note that $\mathcal{H}$ is an additive group. Then the function $f$ defined by $f(\xi):=e^{-\|\xi\|^{2}}(\xi \in \mathcal{H})$ is a positive definite (class) function on $\mathcal{H}$.

Example 2.6 (I. J. Schoenberg [19]). For all $1 \leq p \leq 2$ the function $f_{p}$ defined by $f_{p}(a):=e^{-\|a\|_{p}^{p}}\left(a \in l^{p}\right)$ is a positive definite (class) function on the separable Banach space $l^{p}$.

For more details about positive definite class functions, see [12].

\section{$\S 2.3$. The first characterization}

We now characterize Polish groups of finite type. 
Theorem 2.7. For a Polish group $G$ the following are equivalent:

(i) $G$ is of finite type.

(ii) $G$ is isomorphic as a topological group to a closed subgroup of the unitary group of a finite von Neumann algebra acting on a separable Hilbert space.

(iii) The family $\mathcal{F}$ of continuous positive definite class functions on $G$ generates a neighborhood basis of the identity $e_{G}$ of $G$. That is, for each neighborhood $V$ of the identity, there are functions $f_{1}, \ldots, f_{n} \in \mathcal{F}$ and open sets $\mathcal{O}_{1}, \ldots, \mathcal{O}_{n}$ in $\mathbb{C}$ such that

$$
e_{G} \in \bigcap_{i=1}^{n} f_{i}^{-1}\left(\mathcal{O}_{i}\right) \subset V .
$$

(iv) There exists a positive, continuous, positive definite class function which generates a neighborhood basis of the identity of $G$.

(v) The family $\mathcal{F}$ of continuous positive definite class functions on $G$ separates the identity of $G$ and closed subsets $A$ with $A \not \supset e_{G}$. That is, for each closed subset $A$ with $A \not \supset e_{G}$, there exists $f \in \mathcal{F}$ such that

$$
\sup _{x \in A}|f(x)|<\left|f\left(e_{G}\right)\right| .
$$

(vi) There exists a positive continuous positive definite class function which separates the identity of $G$ and closed subsets $A$ with $A \not \supset e_{G}$.

Proof. (iv) $\Leftrightarrow($ vi $) \Rightarrow(\mathrm{v}) \Rightarrow$ (iii) and (ii) $\Rightarrow$ (i) are trivial.

(iii) $\Rightarrow$ (ii). Since $G$ is first countable, there exists a countable subfamily $\left\{f_{n}\right\}_{n}$ of $\mathcal{F}$ which generates a neighborhood basis of the identity of $G$. Let $\left(\pi_{n}, \xi_{n}, \mathcal{H}_{n}\right)$ be the GNS triple associated to $f_{n}$ and $M_{n}$ be a von Neumann algebra generated by $\pi_{n}(G)$. Since each $M_{n}$ is finite, the direct sum $M:=\bigoplus_{n} M_{n}$ is also finite and acts on a separable Hilbert space $\mathcal{H}:=\bigoplus_{n} \mathcal{H}_{n}$ (see the remark before Lemma 2.4). Put $\pi:=\bigoplus_{n} \pi_{n}$, then $\pi$ is an embedding of $G$ into $\mathcal{U}(M)$. The image of $\pi$ is closed in $\mathcal{U}(M)$, as both $G$ and $\mathcal{U}(M)$ are Polish.

(i) $\Rightarrow$ (iii). Let $\pi$ be an embedding of $G$ into the unitary group of a finite von Neumann algebra $M$. Since each finite von Neumann algebra is the direct sum of countably decomposable finite von Neumann algebras, we can take a family of countably decomposable finite von Neumann algebras $\left\{M_{i}\right\}_{i \in I}$ with $M=\bigoplus_{i \in I} M_{i}$. In this case $\pi$ is also of the form $\pi=\bigoplus_{i \in I} \pi_{i}$, where each $\pi_{i}: G \rightarrow \mathcal{U}\left(M_{i}\right)$ is a continuous group homomorphism. Let $\tau_{i}$ be a faithful normal tracial state on $M_{i}$ and $\left(\rho_{i}, \xi_{i}, \mathcal{H}_{i}\right)$ be its GNS triple as a $C^{*}$-algebra. Here each $\rho_{i}$ is an isomorphism from $M_{i}$ into $\mathbb{B}\left(\mathcal{H}_{i}\right)$ and

$$
\tau_{i}(x)=\left\langle\xi_{i}, \rho_{i}(x) \xi_{i}\right\rangle, \quad x \in M_{i} .
$$


Now set $f_{i}:=\tau_{i} \circ \pi_{i}$. Then each $f_{i}$ is a continuous positive definite class function on $G$ and $\left\{f_{i}\right\}_{i \in I}$ generates a neighborhood basis of the identity $e_{G}$ of $G$.

(iii) $\Rightarrow$ (iv). Let $\left\{f_{n}\right\}_{n}$ be a countable family of continuous positive definite class functions generating a neighborhood basis of the identity of $G$ with $f_{n}\left(e_{G}\right)=1$. Set

$$
f_{n}^{\prime}(g):=e^{\operatorname{Re}\left(f_{n}(g)\right)-1}=e^{-1} \sum_{k=0}^{\infty} \frac{1}{k !}\left[\operatorname{Re}\left(f_{n}(g)\right)\right]^{k}, \quad g \in G .
$$

Then $\left\{f_{n}^{\prime}\right\}_{n}$ is not only a family of continuous positive definite class functions generating a neighborhood basis of the identity of $G$ with $f_{n}^{\prime}\left(e_{G}\right)=1$ but also a family of positive functions. Define a positive, continuous, positive definite class function by $f(g):=\sum_{n} f_{n}^{\prime}(g) / 2^{n}(g \in G)$. It is easy to see that $f$ generates a neighborhood basis of the identity of $G$.

Remark 2.8. The proof of the above theorem is inspired by Theorem 2.1 of S. Gao [10].

Remark 2.9. Popa (Lemma 2.6 of [18]) showed that a Polish group $G$ is of finite type if and only if it is isomorphic to a closed subgroup of the unitary group of a separable $\mathrm{II}_{1}$ factor. Therefore Theorem 2.7 gives a necessary and sufficient condition for a Polish group to be isomorphic to a closed subgroup of the unitary group of a separable $\mathrm{II}_{1}$ factor.

\section{§2.4. SIN-groups and bi-invariant metrics}

To discuss further properties of finite type groups, we consider the notions of SIN-groups, bi-invariant metrics and unitary representability.

A neighborhood $V$ of the identity of a topological group $G$ is called invariant if it is invariant under all inner automorphisms, that is, $g V g^{-1}=V$ for all $g \in G$. A SIN-group is a topological group which has a neighborhood basis of the identity consisting of invariant neighborhoods. Note that a locally compact Hausdorff SINgroup is unimodular.

$A$ bi-invariant metric on a group $G$ is a metric $d$ which satisfies

$$
d(k g, k h)=d(g k, h k)=d(g, h), \quad \forall g, h, k \in G .
$$

It is known that a first countable Hausdorff topological group is SIN if and only if it admits a compatible bi-invariant metric.

As Popa [18] pointed out, one of the most important properties of Polish groups of finite type is the existence of a compatible bi-invariant metric.

Lemma 2.10. Each Polish group of finite type has a compatible bi-invariant metric. In particular, it is SIN. 
Proof. It is enough to show that for every finite von Neumann algebra $M$ acting on a separable Hilbert space $\mathcal{H}$ the unitary group $\mathcal{U}(M)$ has a compatible bi-invariant metric. Let $\tau$ be a faithful normal tracial state on $M$. Then

$$
d(u, v):=\tau\left((u-v)^{*}(u-v)\right)^{1 / 2}, \quad u, v \in \mathcal{U}(M),
$$

is a compatible bi-invariant metric on $\mathcal{U}(M)$.

\section{$\S 2.5$. Unitary representability}

A Hausdorff topological group is called unitarily representable if it is isomorphic as a topological group to a subgroup of the unitary group of a Hilbert space. All locally compact Hausdorff groups are unitarily representable via the left regular representation. It is clear that a Polish group of finite type is necessarily unitarily representable. The following characterization of unitary representability can be found e.g. in Gao [10].

Lemma 2.11. For a Polish group $G$ the following are equivalent.

(i) $G$ is unitarily representable.

(ii) There exists a positive, continuous positive definite function which separates the identity of $G$ and closed subsets $A$ with $A \not \supset e_{G}$.

\section{§2.6. Simple examples}

All of the following examples are well-known. The first three examples are locally compact groups.

Example 2.12. Any compact metrizable group is a Polish group of finite type. This follows from the Peter-Weyl theorem.

Example 2.13. Any abelian second countable locally compact Hausdorff group is a Polish group of finite type. Indeed its left regular representation is an embedding into the unitary group of a Hilbert space and the von Neumann algebra generated by its image is commutative (in particular, finite).

Example 2.14. Any countable discrete group is a Polish group of finite type, for its left regular representation is an embedding into the unitary group of a finite von Neumann algebra.

The following two examples suggest there are few other examples of locally compact groups of finite type. 
Example 2.15. Let $G:=\left\{\left(\begin{array}{ll}x & y \\ 0 & 1\end{array}\right) \in \mathrm{GL}(2, \mathbb{K}) ; x \in \mathbb{K}^{\times}, y \in \mathbb{K}\right\}$ be the $a x+b$ group, where $\mathbb{K}=\mathbb{R}$ or $\mathbb{C}$. By easy computations, we have

$$
\left(\begin{array}{ll}
a & b \\
0 & 1
\end{array}\right)\left(\begin{array}{ll}
x & y \\
0 & 1
\end{array}\right)\left(\begin{array}{ll}
a & b \\
0 & 1
\end{array}\right)^{-1}=\left(\begin{array}{cc}
x & -b x+a y+b \\
0 & 1
\end{array}\right),
$$

so that the conjugacy class $C\left(\left(\begin{array}{ll}x & y \\ 0 & 1\end{array}\right)\right)$ of $\left(\begin{array}{ll}x & y \\ 0 & 1\end{array}\right)$ is

$$
C\left(\left(\begin{array}{ll}
x & y \\
0 & 1
\end{array}\right)\right)= \begin{cases}\left\{\left(\begin{array}{ll}
x & \sharp \\
0 & 1
\end{array}\right) ; \sharp \in \mathbb{K}\right\} & (x \neq 1), \\
\left\{\left(\begin{array}{ll}
1 & \sharp \\
0 & 1
\end{array}\right) ; \sharp \in \mathbb{K}^{\times}\right\} & (x=1, y \neq 0), \\
\left\{\left(\begin{array}{ll}
1 & 0 \\
0 & 1
\end{array}\right)\right\} & (x=1, y=0) .\end{cases}
$$

Thus for each $n \in \mathbb{N}$ there exists a matrix $h_{n} \in G$ such that $h_{n} g_{n} h_{n}^{-1}=\left(\begin{array}{ll}1 & 1 \\ 0 & 1\end{array}\right)$, where $g_{n}:=\left(\begin{array}{cc}1 & 1 / n \\ 0 & 1\end{array}\right)$. Clearly, $g_{n} \rightarrow 1$ and $h_{n} g_{n} h_{n}^{-1} \nrightarrow 1$. This implies that the $a x+b$ group does not admit a compatible bi-invariant metric. Hence it is not of finite type.

Example 2.16. The special linear group $\operatorname{SL}(n, \mathbb{K})(n \geq 2)$ is not of finite type since the map $\left(\begin{array}{ll}a & b \\ 0 & 1\end{array}\right) \mapsto\left(\begin{array}{cc}a & b \\ 0 & a^{-1}\end{array}\right)$ is an embedding of the $a x+b$ group into $\operatorname{SL}(2, \mathbb{K})$. Hence the general linear group $\operatorname{GL}(n, \mathbb{K})(n \geq 2)$ is not of finite type either.

Next we consider abelian groups. Note that an abelian topological group is of finite type if and only if it is unitarily representable.

Example 2.17. Any separable Hilbert space is a Polish group of finite type. This follows from Example 2.5 and Theorem 2.7.

Example 2.18. A separable Banach space $l^{p}(1 \leq p \leq \infty)$ is a Polish group of finite type if and only if $1 \leq p \leq 2$. The "if" part follows from Example 2.6 and Theorem 2.7, but the "only if" part is non-trivial. For details, see [15].

Here is another counterexample.

Example 2.19. The separable Banach space $C[0,1]$ of all continuous functions on the interval $[0,1]$ is a Polish group but not of finite type. Indeed, since every separable Banach space is isometrically isomorphic to a closed subspace of $C[0,1]$, if $C[0,1]$ were of finite type, then any separable Banach space would be a Polish group of finite type. But this is a contradiction to the previous example. 


\section{§2.7. Application to locally compact groups}

It is known that a second countable locally compact group is of finite type if and only if it is a SIN-group (see e.g., Theorem 13.10.5 of J. Dixmier [4]). We give a new proof of this fact using Theorem 2.7. We thank the referee for indicating the above source.

Proposition 2.20. A second countable locally compact Hausdorff group is of finite type if and only if it is SIN.

Proof. Let $G$ be a second countable locally compact Hausdorff SIN-group, $\mu$ be the Haar measure on it and $\lambda$ be its left-regular representation. For each compact invariant neighborhood $U$ of the identity, we define a continuous positive definite function $\varphi_{U}$ on $G$ by

$$
\varphi_{U}(g):=\left\langle\chi_{U}, \lambda(g) \chi_{U}\right\rangle=\mu(U \cap g U), \quad g \in G .
$$

Note that, for each $g, h, x \in G$, we have

$$
h^{-1} x \in U \Leftrightarrow x \in h U=U h \Leftrightarrow x h^{-1} \in U,
$$

and

$$
(g h)^{-1} x \in U \Leftrightarrow x \in g h U=g U h \Leftrightarrow x h^{-1} \in g U .
$$

Also note that a locally compact SIN-group is unimodular. Thus we see that

$$
\begin{aligned}
\varphi_{U}\left(h^{-1} g h\right) & =\left\langle\lambda(h) \chi_{U}, \lambda(g h) \chi_{U}\right\rangle=\int_{G} \chi_{U}\left(h^{-1} x\right) \chi_{U}\left((g h)^{-1} x\right) d \mu(x) \\
& =\int_{G} \chi_{U}\left(x h^{-1}\right) \chi_{g U}\left(x h^{-1}\right) d \mu(x)=\int_{G} \chi_{U}(x) \chi_{g U}(x) d \mu(x) \\
& =\int_{G} \chi_{U}(x) \chi_{U}\left(g^{-1} x\right) d \mu(x)=\varphi_{U}(g),
\end{aligned}
$$

so $\varphi_{U}$ is a class function. It is not hard to check that the family $\left\{\varphi_{U}\right\}_{U}$ generates a neighborhood basis of the identity. This completes the proof by Theorem 2.7.

Remark 2.21. (1) R. V. Kadison and I. Singer [14] proved that every connected locally compact Hausdorff SIN-group is isomorphic as a topological group to a topological group of the form $\mathbb{R}^{n} \times K$, where $K$ is a compact Hausdorff group.

(2) K. Hofmann, S. Morris and M. Stroppel [13] proved that a totally disconnected locally compact Hausdorff group is SIN if and only if it is a strict projective limit of discrete groups. 


\section{$\S 2.8$. A characterization for amenable groups}

Next, we characterize (not necessarily locally compact) amenable Polish groups of finite type. Recall that a Hausdorff topological group $G$ is amenable if $\operatorname{RUCB}(G)$ admits a left-translation invariant positive functional $m \in \operatorname{RUCB}(G)^{*}$ with $m(1)=$ 1 , where $\operatorname{RUCB}(G)$ is the complex Banach space of all right-uniformly continuous bounded functions on $G$. Such an $m$ is called an invariant mean.

Theorem 2.22. A unitarily representable amenable Polish group is of finite type if and only if it is SIN.

Proof. Let $G$ be a unitarily representable amenable Polish SIN-group and let $f$ be a positive, continuous positive definite function on $G$ which separates the identity of $G$ and closed subsets $A$ with $A \not \nexists e_{G}$ (see Lemma 2.11). We may and do assume $f\left(e_{G}\right)=1$. For each $x \in G$, we define a positive function $\Psi_{x, f}: G \rightarrow[0,1]$ by

$$
\Psi_{x, f}(g):=f\left(g^{-1} x g\right), \quad g \in G .
$$

We show that $\Psi_{x, f} \in \operatorname{RUCB}(G)$. Fix an arbitrary $\varepsilon>0$. Since the positive definite function $f$ is right-uniformly continuous, there exists a neighborhood $V$ of $e_{G}$ such that

$$
|f(g)-f(h)|<\varepsilon
$$

holds whenever $g, h \in G$ satisfy $h g^{-1} \in V$. There exists a neighborhood $W$ of $e_{G}$ such that $W=W^{-1}$ and $W \cdot W \subset V$. Since $G$ is SIN, there exists an invariant neighborhood $U$ of $e_{G}$ with $U \subset W$. Let $g, h \in G$ satisfy $h g^{-1} \in U$. By the invariance of $U$, we have $h \in U g=g U$ and therefore $g^{-1} h \in U$. Then

$$
\begin{aligned}
\left(h^{-1} x h\right)\left(g^{-1} x g\right)^{-1} & =h^{-1} x h g^{-1} x^{-1} g \in h^{-1} x U x^{-1} g=h^{-1} U g=U h^{-1} g \\
& =U\left(g^{-1} h\right)^{-1} \subset W \cdot W^{-1} \subset V,
\end{aligned}
$$

which implies

$$
\left|\Psi_{x, f}(h)-\Psi_{x, f}(g)\right|=\left|f\left(h^{-1} x h\right)-f\left(g^{-1} x g\right)\right|<\varepsilon .
$$

Hence $\Psi_{x, f}$ is right-uniformly continuous and we have $\Psi_{x, f} \in \operatorname{RUCB}(G)_{+}$. Let $m \in \operatorname{RUCB}(G)^{*}$ be an invariant mean. Put

$$
\psi_{f}(x):=m\left(\Psi_{x, f}\right), \quad x \in G .
$$

Then $\psi_{f}(x)$ is clearly a positive, positive definite class function on $G$ with $\psi_{f}\left(e_{G}\right)$ $=1$. We show that $\psi_{f}$ is continuous. Since $m$ is continuous, it suffices to show that 
$G \ni x \mapsto \Psi_{x, f} \in \operatorname{RUCB}(G)_{+}$is continuous. Let $x, y \in G$. By Krein's inequality,

$$
\begin{aligned}
\left\|\Psi_{x, f}-\Psi_{y, f}\right\|^{2} & =\sup _{g \in G}\left|f\left(g^{-1} x g\right)-f\left(g^{-1} y g\right)\right|^{2} \\
& \leq 2 \sup _{g \in G}\left|1-\operatorname{Re} f\left(g^{-1} y x^{-1} g\right)\right|=2 \sup _{g \in G}\left|1-f\left(g^{-1} y x^{-1} g\right)\right| .
\end{aligned}
$$

Fix $\varepsilon>0$. Since $f$ is right-uniformly continuous, there exists an invariant neighborhood $V$ of $e_{G}$ such that $|f(x)-f(y)|<\varepsilon$ for $x, y \in G$ with $y x^{-1} \in V$. Then for $x, y \in G$ with $y x^{-1} \in V$, we have $g^{-1} y x^{-1} g \in g^{-1} V g=V$. Consequently,

$$
\left|1-f\left(g^{-1} y x^{-1} g\right)\right|=\left|f\left(e_{G}\right)-f\left(g^{-1} y x^{-1} g\right)\right|<\varepsilon .
$$

Hence

$$
\left\|\Psi_{x, f}-\Psi_{y, f}\right\|^{2} \leq 2 \varepsilon .
$$

Therefore $G \ni x \mapsto \Psi_{x, f} \in \operatorname{RUCB}(G)_{+}$is continuous, hence so is $\psi_{f}$. We next show that $\psi_{f}$ separates the identity of $G$ and closed subsets $A$ with $A \not \supset e_{G}$. Fix such an $A$. Since $A^{c}=G \backslash A$ is an open neighborhood of $e_{G}$, there exists an open invariant neighborhood $V$ of $e_{G}$ contained in $A^{c}$. Then $A \subset V^{c}$ and $e_{G} \notin V^{c}$. Since $f$ separates $e_{G}$ and $V^{c}$, we have

$$
\delta:=\sup _{g \in V^{c}}|f(g)|<1 .
$$

It then follows, by the invariance of $V^{c}$, that for $x \in V^{c}$,

$$
\left\|\Psi_{x, f}\right\|=\sup _{g \in G}\left|f\left(g^{-1} x g\right)\right| \leq \sup _{g \in V^{c}}|f(g)| \leq \delta,
$$

which implies

$$
\sup _{x \in A}\left|\psi_{f}(x)\right| \leq \sup _{x \in V^{c}}\left|\psi_{f}(x)\right|=\sup _{x \in V^{c}}\left|m\left(\Psi_{x, f}\right)\right| \leq \sup _{x \in V^{c}}\left\|\Psi_{x, f}\right\| \leq \delta<1 .
$$

Therefore $\psi_{f}$ separates $A$ and $e_{G}$. This completes the proof by Theorem 2.7.

Remark 2.23. The above proof is inspired by the proof of Theorem 2.13 of J. Galindo [9].

\section{§3. More examples of finite type groups}

In this section we will give other examples of Polish groups of finite type. To construct them we start not from finite von Neumann algebras, but from semifinite ones, say of type $\mathrm{I}_{\infty}$ or of type $\mathrm{II}_{\infty}$. At the end of this section we also review other known examples of Polish groups of finite type. 


\section{$\S 3.1 . L^{2}$-unitary groups $\mathcal{U}(M)_{2}$}

Let $M$ be a semifinite von Neumann algebra on a Hilbert space $\mathcal{H}$ equipped with a normal faithful semifinite trace $\tau$. A densely defined, closed operator $T$ on $\mathcal{H}$ is said to be affiliated to $M$ if $u T u^{*}=T$ for all $u \in \mathcal{U}\left(M^{\prime}\right)$. Denote by $\bar{M}$ the set of all densely defined, closed operators on $\mathcal{H}$ which are affiliated to $M$. Recall that $L^{2}(M, \tau)$ is a Hilbert space completion of the space $\mathfrak{n}_{\tau}:=\left\{x \in M ; \tau\left(x^{*} x\right)<\infty\right\}$ with the inner product

$$
\langle x, y\rangle:=\tau\left(x^{*} y\right), \quad x, y \in \mathfrak{n}_{\tau} .
$$

We define $\|x\|_{2}:=\tau\left(x^{*} x\right)^{1 / 2}$ for $x \in L^{2}(M, \tau)$.

Definition 3.1. We call $\mathcal{U}(M)_{2}:=\left\{u \in \mathcal{U}(M) ; 1-u \in L^{2}(M, \tau)\right\}$ the $L^{2}$-unitary group of $(M, \tau)$.

Note that when $M$ is not a factor, $\mathcal{U}(M)_{2}$ also depends on the choice of $\tau$. We will show the following theorem.

Theorem 3.2. Let $M$ be a separable semifinite von Neumann algebra with a normal faithful semifinite trace $\tau$. Then $\mathcal{U}(M)_{2}$ is a Polish group of finite type, where the topology is determined by the metric

$$
d(u, v):=\|u-v\|_{2}, \quad u, v \in \mathcal{U}(M)_{2} .
$$

To prove the theorem, we need some preparations. We consider $M$ to be represented on $\mathcal{H}=L^{2}(M, \tau)$ by left multiplication. Recall that a closed operator $T \in \bar{M}$ on $L^{2}(M, \tau)$ is called $\tau$-measurable if for any $\varepsilon>0$, there exists a projection $p \in M$ with $\operatorname{ran}(p) \subset \operatorname{dom}(T)$ and $\tau(1-p)<\varepsilon$. Note that $L^{2}(M, \tau)$ can be identified with the set of closed, densely defined and $\tau$-measurable operators $T$ such that

$$
\|T\|_{2}^{2}:=\tau\left(|T|^{2}\right)=\int_{0}^{\infty} \lambda^{2} d \tau(e(\lambda))<\infty,
$$

where $e(\cdot)$ is the spectral measure of $|T|=\left(T^{*} T\right)^{1 / 2}$ and $T=u|T|$ is the polar decomposition of $T$ (for more details about noncommutative integration, see Vol. II of $[20])$.

Lemma 3.3. Let $M$ be a semifinite von Neumann algebra with a normal faithful semifinite trace $\tau$. Then $\mathcal{U}(M)_{2}$ is a topological group.

Proof. This can be shown directly, using the equalities

$$
\left\|x^{*}\right\|_{2}=\|x\|_{2}, \quad\|u x v\|_{2}=\|x\|_{2},
$$

for all $x \in L^{2}(M, \tau)$ and $u, v \in \mathcal{U}(M)$. 
Lemma 3.4. Let $M$ be a semifinite von Neumann algebra with a normal faithful semifinite trace $\tau$. Let $U$ be a densely defined closed $\tau$-measurable operator on $L^{2}(M, \tau)$ affiliated to $M$. Then $\operatorname{dom}(U) \cap M$ is dense in $L^{2}(M, \tau)$.

Proof. Let $\varepsilon>0$. Let $\xi \in L^{2}(M, \tau)$. Since $M \cap L^{2}(M, \tau)$ is dense, there exists $\xi_{0} \in M \cap L^{2}(M, \tau)$ such that $\left\|\xi-\xi_{0}\right\|_{2}<\varepsilon$. On the other hand, the measurability of $U$ implies the existence of an increasing sequence $\left\{p_{n}\right\}_{n=1}^{\infty}$ of projections in $M$ such that $p_{n} L^{2}(M, \tau) \subset \operatorname{dom}(U)$ for all $n$ and $p_{n} \nearrow 1$ strongly. Therefore there exists $n_{0} \in \mathbb{N}$ such that

$$
\left\|\xi_{0}-p_{n_{0}} \xi_{0}\right\|_{2}<\varepsilon
$$

By the choice of $\xi_{0}, p_{n_{0}} \xi_{0} \in \operatorname{dom}(U) \cap M$ and

$$
\left\|\xi-p_{n_{0}} \xi_{0}\right\|_{2} \leq\left\|\xi-\xi_{0}\right\|_{2}+\left\|\xi_{0}-p_{n_{0}} \xi_{0}\right\|_{2} \leq \varepsilon+\varepsilon=2 \varepsilon \text {. }
$$

Since $\varepsilon$ is arbitrary, it follows that $\operatorname{dom}(U) \cap M$ is dense in $L^{2}(M, \tau)$.

Lemma 3.5. Let $M$ be a semifinite von Neumann algebra with a normal faithful semifinite trace $\tau$. Then $d$ is a complete metric on $\mathcal{U}(M)_{2}$.

Proof. Suppose $\left\{u_{n}\right\}_{n=1}^{\infty}$ is a $d$-Cauchy sequence in $\mathcal{U}(M)_{2}$. Since $L^{2}(M, \tau)$ is complete, there exists $V \in L^{2}(M, \tau)$ such that $\left\|\left(1-u_{n}\right)-V\right\|_{2} \rightarrow 0$. Define $U:=1-V$. Then $\left\|U-u_{n}\right\|_{2} \rightarrow 0$. We show that $U$ is bounded and moreover $U \in \mathcal{U}(M)_{2}$. Since $U$ is closed and $\operatorname{dom}(U) \cap M$ is dense by Lemma 3.4, to prove the boundedness of $U$ it suffices to show that $U$ is isometric on $\operatorname{dom}(U) \cap M$. Let $\xi \in \operatorname{dom}(U) \cap M$. Since $\xi$ is bounded, we have

$$
\begin{aligned}
\left\|\left(U-u_{n}\right) \xi\right\|_{2}^{2} & =\tau\left(\xi^{*}\left(U-u_{n}\right)^{*}\left(U-u_{n}\right) \xi\right)=\tau\left(\left(U-u_{n}\right) \xi \xi^{*}\left(U-u_{n}\right)^{*}\right) \\
& \leq\|\xi\|^{2} \tau\left(\left(U-u_{n}\right)\left(U-u_{n}\right)^{*}\right)=\|\xi\|^{2}\left\|U-u_{n}\right\|_{2}^{2} \rightarrow 0,
\end{aligned}
$$

which implies

$$
\|U \xi\|_{2}=\lim _{n \rightarrow \infty}\left\|u_{n} \xi\right\|_{2}=\|\xi\|_{2}
$$

for all $\xi \in \operatorname{dom}(U) \cap M$. Therefore $\left.U\right|_{\operatorname{dom}(U) \cap M}$ is isometric and $U$ is bounded. Since $\left\|U^{*}-u_{n}^{*}\right\|_{2}=\left\|U-u_{n}\right\|_{2}, U^{*}$ is an isometry too, which means $U$ is a unitary. Finally, it is clear that $U=1-V \in \mathcal{U}(M)_{2}$.

Proof of Theorem 3.2. Since $M$ is separable, the separability of $\mathcal{U}(M)_{2}$ follows from the separability of $L^{2}(M, \tau)$. Therefore by Lemma $3.5, \mathcal{U}(M)_{2}$ is a Polish group. By Schoenberg's theorem (see Example 2.5),

$$
\varphi(u):=e^{-\|1-u\|_{2}^{2}}, \quad u \in \mathcal{U}(M)_{2},
$$


is a continuous, positive definite class function on $\mathcal{U}(M)_{2}$. It is easy to see that $\varphi$ generates a neighborhood basis of the identity of $\mathcal{U}(M)_{2}$. Therefore the claim follows from Theorem 2.7 .

Remark 3.6. $\mathcal{U}(M)_{2}^{\prime \prime}=M$.

Proof. Clearly $\mathcal{U}(M)_{2}^{\prime \prime} \subset M$. Let $p$ be a finite projection in $M$. Then $2 p \in L^{2}(M, \tau)$ and $1-2 p \in \mathcal{U}(M)_{2}$. Therefore $p \in \mathcal{U}(M)_{2}^{\prime \prime}$. Since $M$ is semifinite, $M$ is generated by finite projections. Therefore $\mathcal{U}(M)_{2}^{\prime \prime}=M$.

When $M=\mathbb{B}(\mathcal{H}), \mathcal{U}(M)_{2}$ is the well-known example of a Hilbert-Lie group and is denoted by $\mathcal{U}(\mathcal{H})_{2}$.

\section{§3.2. Nonisomorphic properties of $\mathcal{U}(M)_{2}$}

J. Feldman [7] gave a complete description of group isomorphisms between the unitary groups of type $\mathrm{II}_{1}$ von Neumann algebras. In particular, in the proof of Theorem 4 of [7], he uses the following simple observation: if $p$ is a projection in a von Neumann algebra $M$, then $u_{p}:=1-2 p$ is a self-adjoint unitary in $M$. Using this correspondence, he deduces that a group isomorphism $\pi: \mathcal{U}\left(M_{1}\right) \rightarrow \mathcal{U}\left(M_{2}\right)$ between type $\mathrm{II}_{1}$ von Neumann algebras $M_{1}, M_{2}$ induces an order isomorphism between their projection lattices, thereby proving that $\pi$ lifts to a ring *-isomorphism $\bar{\pi}: M_{1} \rightarrow M_{2}$ (which may not preserve scalar multiplication) such that

$$
\bar{\pi}(u)=\theta(u) \pi(u) \quad \text { for all } u \in \mathcal{U}\left(M_{1}\right),
$$

where $\theta$ is a multiplicative map from $\mathcal{U}\left(M_{1}\right)$ to $Z\left(\mathcal{U}\left(M_{2}\right)\right)$. Let $\mathcal{H}$ be an infinitedimensional Hilbert space. Using his idea, we show that when $M$ is a $\mathrm{II}_{\infty}$ factor and $N$ is a finite von Neumann algebra, then $\mathcal{U}(M)_{2}, \mathcal{U}(\mathcal{H})_{2}$ and $\mathcal{U}(N)$ are mutually nonisomorphic. In this subsection, no separability assumptions are required.

Proposition 3.7. Let $M$ be a $I I_{\infty}$ factor. Then $\mathcal{U}(M)_{2}$ is not isomorphic to $\mathcal{U}(\mathcal{H})_{2}$.

Proof. Let $\tau$ be a normal faithful semifinite trace on $M$, and $\operatorname{Tr}$ be the usual operator trace on $\mathcal{H}$. We denote the corresponding trace 2-norms by $\|\cdot\|_{2, \tau}$ and $\|\cdot\|_{2, \operatorname{Tr}}$, respectively. For contradiction, suppose there exists a topological group isomorphism $\varphi: \mathcal{U}(M)_{2} \rightarrow \mathcal{U}(\mathcal{H})_{2}$. Let $p$ be a nonzero finite-rank projection in $\mathbb{B}(\mathcal{H})$. Then $1-2 p \in \mathcal{U}(\mathcal{H})_{2}$ and let

$$
q:=\frac{1}{2}\left(1-\varphi^{-1}(1-2 p)\right) .
$$

It is easy to see that $q \in L^{2}(M, \tau)$ is a nonzero finite projection in $M$. Let $k \in \mathbb{N}$. 
Since $M$ is a $\mathrm{II}_{\infty}$ factor, there exists a projection $0<q_{k} \leq q$ in $M$ such that $\lim _{k \rightarrow \infty} \tau\left(q_{k}\right)=0$. Define $p_{k}:=\frac{1-\varphi\left(1-2 q_{k}\right)}{2}$. Since

$$
\left\|q_{k}\right\|_{2, \tau}^{2}=\tau\left(q_{k}\right) \rightarrow 0 \quad(k \rightarrow \infty)
$$

we have $1-2 q_{k} \rightarrow 1$ in $\mathcal{U}(M)_{2}$, which in turn means

$$
1-2 p_{k}=\varphi\left(1-2 q_{k}\right) \rightarrow \varphi(1)=1 \text { in } \mathcal{U}(\mathcal{H})_{2} .
$$

However, since the topology of $\mathcal{U}(\mathcal{H})_{2}$ is given by the operator trace 2-norm,

$$
2 \leq\left\|2 p_{k}\right\|_{2, \operatorname{Tr}}=\left\|1-\left(1-2 p_{k}\right)\right\|_{2, \operatorname{Tr}} \rightarrow 0 \quad(k \rightarrow \infty) .
$$

This is clearly a contradiction. Therefore $\mathcal{U}(M)_{2} \neq \mathcal{U}(\mathcal{H})_{2}$.

Proposition 3.8. Let $M$ be a type $I_{\infty}$ or type $I I_{\infty}$ factor, and $N$ be a finite von Neumann algebra. Then $\mathcal{U}(M)_{2}$ is not isomorphic onto $\mathcal{U}(N)$.

Proof. Let $\tau$ be a normal faithful semifinite trace on $M$. Let $u \in Z\left(\mathcal{U}(M)_{2}\right)$, so $u$ commutes with every element in $\mathcal{U}(M)_{2}$. Then $p \in M, u(1-2 p)=(1-2 p) u$ for any finite projection $p \in M$. Therefore $u$ commutes with all finite projections in $M$. Since $M$ is generated by its finite projections, it follows that $u \in Z(M)=\mathbb{C} 1$. Since $u-1 \in L^{2}(M, \tau)$, this forces $u=1$. Therefore the center of $\mathcal{U}(M)_{2}$ is $\{1\}$, while the center of $\mathcal{U}(N)$ contains $\mathbb{C} 1$.

Remark 3.9. We thank the referee for communicating the above simple proof and calling our attention to [7].

\section{§3.3. Other known examples}

The class $\mathscr{U}_{\text {fin }}$ has not yet been studied well. However, there are some known examples other than the ones presented in $\S 2.6$.

Example 3.10 (Normalizer groups $\mathcal{N}_{M}(A)$ and $\mathcal{N}(E)$ ). Let $A$ be an abelian von Neumann subalgebra of a separable $\mathrm{II}_{1}$ factor $M$. The normalizer group $\mathcal{N}_{M}(A)$ of $A$, defined by

$$
\mathcal{N}_{M}(A):=\left\{u \in \mathcal{U}(M) ; u A u^{*}=A\right\},
$$

is clearly a strongly closed subgroup of $\mathcal{U}(M)$ and hence belongs to $\mathscr{U}_{\text {fin }}$. This group has drawn much attention of specialists, especially when $A$ is maximal abelian and $\mathcal{N}_{M}(A)$ generates $M$ as a von Neumann algebra. In that case, $A$ is called a Cartan subalgebra. Similarly, the normalizer group $\mathcal{N}(E)$ for a normal faithful conditional expectation $E: M \rightarrow N$ onto a von Neumann subalgebra $N$,

$$
\mathcal{N}(E):=\left\{u \in \mathcal{U}(M) ; u E(x) u^{*}=E\left(u x u^{*}\right) \text { for all } x \in M\right\},
$$

is also of finite type. 
Example 3.11 (The full group $[\mathcal{R}]$ ). Let $\mathcal{R}$ be a $\mathrm{II}_{1}$ countable equivalence relation on a standard probability space $(X, \mu)$. A. Furman $[8, \S 2]$ showed that the full group $[\mathcal{R}]$ equipped with so-called uniform topology is a Polish group of finite type.

\section{$\S 4$. Hereditary properties of finite type groups}

In this section, we discuss permanence properties of the class $\mathscr{U}_{\text {fin }}$ under several algebraic operations. In summary, we will observe the following permanence properties of finite type groups:

\begin{tabular}{cc}
\hline Operation & $\mathscr{U}_{\text {fin }} ?$ \\
\hline Closed subgroup $H<G$ & YES \\
Countable direct product $\prod_{n \geq 1} G_{n}$ & YES \\
Semidirect product $G \rtimes H$ & NO \\
Quotient $G / N$ & NO \\
Extension $1 \rightarrow N \rightarrow G \rightarrow K \rightarrow 1$ & NO \\
Projective $\operatorname{limit} \underset{\leftarrow}{\lim } G_{n}$ & YES \\
\hline
\end{tabular}

As can be seen from the above table, the finiteness property is delicate and can easily break down under natural operations.

Remark 4.1 (Ultraproducts of metric groups). Let $\left\{\left(G_{n}, d_{n}\right)\right\}_{n=1}^{\infty}$ be a sequence of finite type Polish groups with a compatible bi-invariant metric. It is not difficult to show that the ultraproduct $\left(G_{\omega}, d_{\omega}\right)$ of $\left\{\left(G_{n}, d_{n}\right)\right\}_{n=1}^{\infty}$ along a free ultrafilter $\omega \in \beta \mathbb{N} \backslash \mathbb{N}$ is a completely metrizable topological group of finite type, but not Polish in general. We will discuss topological groups which are embeddable into the unitary group of a (not necessarily separable) finite von Neumann algebra elsewhere.

\section{$\S 4.1$. Closed subgroups and countable direct products}

It is clear the class $\mathscr{U}_{\text {fin }}$ is closed under taking a closed (or even $G_{\delta}$ ) subgroup. Since a countable direct sum of separable finite von Neumann algebras is again separable and finite, the class $\mathscr{U}_{\text {fin }}$ is closed under countable direct products.

\section{$\S 4.2$. Extensions and semidirect products}

The class $\mathscr{U}_{\text {fin }}$ is not closed under extensions or semidirect products:

Proposition 4.2. There exists a Polish group $G$ not of finite type, which has a closed normal subgroup $N$ such that $N$ and the quotient group $G / N$ are of finite type. 
Proof. Let $G$ be the $a x+b$ group (see Example 2.15). Since $G$ does not have a compatible bi-invariant metric, it is not of finite type. On the other hand, $G$ can be written as a semidirect product $G=\mathbb{K} \rtimes \mathbb{K}^{\times}$, where $\mathbb{K}^{\times}$acts on $\mathbb{K}$ by multiplication. Therefore the exact sequence

$$
0 \rightarrow \mathbb{K} \rightarrow G \rightarrow \mathbb{K}^{\times} \rightarrow 1
$$

gives a counterexample for the extension case.

Note that the above example also shows that the class $\mathscr{U}_{\text {fin }}$ is not closed under semidirect products.

\section{$\S 4.3$. Quotients}

The class $\mathscr{U}_{\text {fin }}$ is not closed under quotients:

Proposition 4.3. There exists an abelian Polish group $G$ of finite type and a closed subgroup $N$ such that the quotient $G / N$ is not of finite type.

Proof. Consider the separable Banach space $A:=l^{3}$ as an additive Polish group. As we saw in Example 2.18, $l^{p}(1 \leq p \leq \infty)$ is unitarily representable if and only if $1 \leq p \leq 2$. On the other hand, every separable Banach space is isomorphic to a quotient of $\ell^{1}$ (see e.g., Theorem 5.1 of [6]). In particular, although not of finite type, $A=\ell^{3}$ is a quotient of $G:=\ell^{1}$ by a closed subgroup.

Remark 4.4. Note that even for abelian Polish groups, the situation can be worst possible. It is known (Chapter 4 of [2]) that there exists an abelian Polish group $A$ which has no nontrivial unitary representation. Such a group is called strongly exotic. On the other hand, S. Gao and V. Pestov [11] proved that any abelian Polish group is a quotient of $\ell^{1}$ by a closed subgroup $N$. Therefore, strongly exotic groups are also quotients of finite type Polish groups.

\section{§4.4. Projective limits}

The class $\mathscr{U}_{\text {fin }}$ is closed under projective limits:

Proposition 4.5. Let $\left\{G_{n}, j_{m, n}: G_{m} \rightarrow G_{n}(n \leq m)\right\}_{n, m=1}^{\infty}$ be a projective system of Polish groups of finite type. Then $G=\lim _{\longleftarrow} G_{n}$ is a Polish group of finite type.

Proof. Since the connecting maps $j_{m, n}$ are continuous, it is clear that $G$ can be seen as a closed subgroup of $\prod_{n \in \mathbb{N}} G_{n}$. Since the finiteness property passes to direct products, $\prod_{n \in \mathbb{N}} G_{n}$ is also a Polish group of finite type. Therefore its closed subgroup $G$ is also Polish of finite type. 


\section{$\S 5$. Some questions}

Finally let us discuss some questions to which we do not have answers at this stage. Let $\mathscr{U}_{\text {inv }}$ denote the class of Polish groups with a compatible bi-invariant metric. As we saw in Example 2.18, $\mathscr{U}_{\text {inv }}$ is strictly larger than $\mathscr{U}_{\text {fin }}\left(l^{3}\right.$ is in $\mathscr{U}_{\text {inv }}$ but not in $\mathscr{U}_{\text {fin }}$ ). Therefore the unitary representability is indispensable (this was also pointed out by Popa). Furthermore, there exists a more interesting example. Recently L. van den Dries and S. Gao [5] constructed a Polish group $G$ with a compatible bi-invariant metric, which does not have Lie sums (see [5] for the definition). On the other hand, we proved in [1] that each group in $\mathscr{U}_{\text {fin }}$ has Lie sums. Thus $G$ is not of finite type. Therefore it would be desirable to consider the following questions (the latter was posed by Popa $[18, \S 6.5]$ ):

Question 5.1. Is van den Dries-Gao's Polish group unitarily representable?

Question 5.2 (Popa). Is every unitarily representable Polish SIN-group of finite type?

Hopefully Theorem 2.7 will play a role in solving the above questions. Also, since $l^{p}$ belongs to $\mathscr{U}_{\text {fin }}$ if and only if $1 \leq p \leq 2$, it is worth considering

Question 5.3. Let $\mathcal{H}$ be a separable infinite-dimensional Hilbert space. Does $\mathcal{U}(\mathcal{H})_{p}:=\left\{u \in \mathcal{U}(\mathcal{H}) ; 1-u \in S^{p}(\mathcal{H})\right\}$ belong to $\mathscr{U}_{\text {fin }}$ for some $1 \leq p<2$ ? Here $S^{p}(\mathcal{H})$ denotes the space of Schatten $p$-class operators.

Finally, let us recall another candidate for a counterexample to Question 5.2. A finite von Neumann algebra $N$ equipped with a normal faithful tracial state $\tau$ is said to have property (T) if for each $\varepsilon>0$, there exists a finite set $\mathcal{F} \subset N$ and $\delta>0$ such that whenever $\varphi: N \rightarrow N$ is a unital completely positive $\tau$-preserving map satisfying $\|\varphi(x)-x\|_{2}<\delta$ for all $x \in \mathcal{F}$, then $\|\varphi(a)-a\|_{2} \leq \varepsilon\|a\|$ for all $a \in N$.

Let $M$ be a separable $\mathrm{II}_{1}$ factor with property $(\mathrm{T})$, and $\operatorname{Aut}(M)$ be the Polish group of all *-automorphisms of $M$ equipped with the pointwise $\|\cdot\|_{2}$-convergence topology. Due to property $(\mathrm{T})$, this topology coincides with the topology of uniform $\|\cdot\|_{2}$-convergence on the closed unit ball $M_{1}$. Since the latter topology is given by the bi-invariant metric $d$ defined by

$$
d(\alpha, \beta):=\sup _{x \in M_{1}}\|\alpha(x)-\beta(x)\|_{2}, \quad \alpha, \beta \in \operatorname{Aut}(M),
$$

$\operatorname{Aut}(M)$ is a Polish SIN-group. By considering the standard representation, $\operatorname{Aut}(M)$ is unitarily representable as well. Therefore it would be interesting to 
check if $\operatorname{Aut}(M)$ is actually of finite type or not. (Added in proof: After this article was accepted for publication, the affirmative answer to the above question was communicated to us by Uffe Haagerup.)

\section{Acknowledgements}

We would like to express our thanks to Professors Asao Arai, Uffe Haagerup, Izumi Ojima and Konrad Schmüdgen for important comments, discussions and support. We also thank Takahiro Hasebe, Ryo Harada, Kazuya Okamura and Dr. Hayato Saigo for discussions in a seminar and for continual interest in our work. The second named author thanks Abel Stolz for interesting discussions. The final version of the paper was prepared during the conference "Von Neumann algebras and ergodic theory of group actions 2011" at Institut Henri Poincaré. We thank the organizers, Professors Damien Gaboriau, Sorin Popa and Stefaan Vaes for fruitful discussions, and also Professor Jesse Peterson for informing us about his recent joint work with Popa on cocycle superrigidity and the class $\mathscr{U}_{\text {fin }}$. Last but not least, we thank the anonymous referee for literature indications and suggesting some simpler proofs. Both the authors are supported by research fellowships of the Japan Society for the Promotion of Science for Young Scientists.

\section{References}

[1] H. Ando and Y. Matsuzawa, Lie group-Lie algebra correspondences of unitary groups in finite von Neumann algebras, Hokkaido Math. J. 41 (2012), 31-99. Zbl pre06011490

[2] W. Banaszczyk, Additive subgroups of topological vector spaces, Lecture Notes in Math. 1466, Springer, Berlin, 1991. Zbl 0743.46002 MR 1119302

[3] D. Beltiţă, Lie theoretic significance of the measure topologies associated with a finite trace, Forum Math. 22 (2010), 241-253. Zbl 1188.22002 MR 2607563

[4] J. Dixmier, $C^{*}$-algebras, North-Holland Math. Library 15, North-Holland, 1977. Zbl 0372.46058 MR 0458185

[5] L. van den Dries and S. Gao, A Polish group without Lie sums, Abh. Math. Sem. Hamburg 79 (2009) 135-147. Zbl 1181.22006 MR 2541347

[6] M. Fabian, P. Habala, P. Hájek, V. Montesinos and V. Zizler, Banach space theory: The basis for linear and nonlinear analysis, CMS Books Math., Springer, New York, 2011. Zbl 1229.46001 MR 2766381

[7] J. Feldman, Isomorphisms of finite type II rings of operators, Ann. of Math. (2) 63 (1956), 565-571. Zbl 0070.34303 MR 0078641

[8] A. Furman, On Popa's cocycle superrigidity theorem, Int. Math. Res. Notices 2007, no. 19, art. ID rnm073, 46 pp. Zbl 1134.46043 MR 2359545

[9] J. Galindo, On group and semigroup compactifications of topological groups, lecture notes for "Topological groups: Introduction to dynamical systems", Madrid, April 3-5, 2008.

[10] S. Gao, Unitary group actions and Hilbertian Polish metric spaces. in Logic and its applications, Contemp. Math. 380, Amer. Math. Soc. Providence, RI, 2005, 53-72. Zbl 1085.54026 MR 2167574 
[11] S. Gao and V. Pestov, On a universality property of some abelian Polish groups, Fund. Math. 179 (2003), 1-15. Zbl 1057.22003 MR 2028923

[12] T. Hirai and E. Hirai, Positive definite class functions on a topological group and characters of factor representations, J. Math. Kyoto Univ. 45 (2005), 355-379. Zbl 1096.22005 MR 2161697

[13] K. Hofmann, S. Morris and M. Stroppel, Varieties of topological groups, Lie groups and SIN-groups, Colloq. Math. 70 (1996), 151-163. Zbl 0853.22001 MR 1380372

[14] R. V. Kadison and I. M. Singer, Some remarks on representations of connected groups, Proc. Nat. Acad. Sci. USA 38 (1952), 419-423. Zbl 0046.25202 MR 0048456

[15] M. Megrelishvili, Reflexively but not unitarily representable topological groups, Topology Proc. 25 (2000), 615-625. Zbl 1026.22006 MR 1925711

[16] J. Peterson and T. Sinclair, On cocycle superrigidity for Gaussian actions, arXiv:0910.3958, 2009.

[17] E. Nelson, Notes on non-commutative integration, J. Funct. Anal. 15 (1974), 103-116. Zbl 0292.46030 MR 0355628

[18] S. Popa, Cocycle and orbit equivalence superrigidity for malleable actions of $w$-rigid groups, Invent. Math. 170 (2007), 243-295. Zbl 1131.46040 MR 2342637

[19] I. J. Schoenberg, Metric spaces and positive definite functions, Trans. Amer. Math. Soc. 44 (1938), 522-536. Zbl 0019.41502 MR 1501980

[20] M. Takesaki, Theory of operator algebras, I-III, Springer, Berlin, 2002-2003. Zbl 0436.46043(I) Zbl 1059.46031(II) Zbl 1059.46032(III) MR 1873025(I) MR 1943006(II) MR 1943007(III) 